\title{
Ocorrência de Dípteros Muscóides (Calliphoridae) no Município de Vassouras, RJ
}

\author{
Raquel S. Macedo \\ Universidade Severino Sombra, Centro de Ciências Exatas, \\ Tecnológicas e da Natureza, Laboratório de Insetos Vetores, na \\ Unidade Ensino, Pesquisa e Extensão Prof. Antonio o. Izolani, \\ Vassouras, RJ \\ Vinicius M. Carraro \\ Universidade Severino Sombra, Centro de Ciências Exatas, \\ Tecnológicas e da Natureza, Laboratório de Insetos Vetores, na \\ Unidade Ensino, Pesquisa e Extensão Prof. Antonio O. Izolani, \\ Vassouras, RJ \\ Cleber B. Espindola \\ Universidade Severino Sombra, Centro de Ciências Exatas,
ecnológicas e da Natureza, Laboratório de Insetos Vetores, na
Unidade Ensino, Pesquisa e Extensão Prof. Antonio o. Izolani,
Vassouras, RJ \\ Marise M. O. Cabral \\ Universidade Severino Sombra, Centro de Ciências Exatas, \\ Tecnológicas e da Natureza, Laboratório de Insetos Vetores, na \\ Unidade Ensino, Pesquisa e Extensão Prof. Antonio O. Izolani, \\ Instituto Oswaldo Cruz, Fundação Oswaldo Cruz, Laboratório de \\ Diptera, Av. Brasil 4365, 21040-900 Rio de Janeiro, RJ, Brasil
}

Resumo: Com este estudo pretendeu-se avaliar a ocorrência de dípteros muscóoides em dois ambientes (rural e urbano) do município de Vassouras - RJ. As amostragens foram realizadas entre outubro/2005 e novembro/2006. Foram coletadas 2185 moscas e a família Calliphoridae apresentou o maior número de indivíduos (1421), seguida da família Sarcophagidae com 743 indivíduos e da familia Muscidae, com apenas 21 individuos coletados. No ambiente rural, as espécies numericamente mais importantes foram Chrysomya megacephala (25,92\%) e Phaenicia eximia (13,15\%). No ambiente urbano, as espécies numericamente mais importantes foram C. megacephala $(20,63 \%)$ e C. albiceps $(14,46 \%)$. Os sarcofagídeos totalizaram um percentual de $28,45 \%$ no ambiente rural e 39,29\% no ambiente urbano. A família Muscidae apresentou menos de $1 \%$ dos indivíduos coletados em ambas às áreas de amostragem, e identificados como M. domestica. Os resultados apontaram C. megacephala como a espécie mais bem representada neste levantamento.

Palavras-chave: Calliphoridae. Diptera. Levantamento populacional. 


\title{
The Occurrence of Muscoidea Diptera (Calliphoridae) in the Vassouras City, Rj.
}

\begin{abstract}
The study aimed to evaluate the occurrence of muscoid flies in two environments (rural and urban) in the Vassouras city, RJ. The samples were done between October 2005 and November 2006. In total, 2185 flies were collected. The Calliphoridae family presented the highest number of individuals (1421) followed by the Sarcophagidae family with 743 and Muscidae with only 21. In the rural environment, the numerically most important species were Chrysomya megacephala (25.92\%) and Phaenicia eximia (13.15\%). In the urban environment, C. megacephala (20.63\%) and C. albiceps (14.46\%) were the numerically most important species. The Sarcophagidae family accounted for $28.45 \%$ in the rural and $39.29 \%$ in the urban environment. The Muscidae family accounted for less than 1\% of the individuals collected in both sampling areas; they were identified as $M$. domestica. The results showed that $C$. megacephala was the species most represented in this survey.
\end{abstract}

Keywords: Calliphoridae. Diptera. Population survey.

\section{Introdução}

A ordem Díptera engloba as moscas, insetos de grande importância sanitária, pois são vetores biológicos e mecânicos de agentes patogênicos de diversas enfermidades que afetam o homem e animais domésticos, além de causarem incômodos e produzirem as miíases (Robinson 1996). As moscas provavelmente desenvolveram a sinantropização desde o início da jornada evolutiva dos hominídeos, aproveitando os depósitos de restos alimentares, carcaças de animais e fezes acumuladas. Com o início da domesticação dos animais também se associaram várias espécies de moscas coprófagas e sarcossaprófagas (Robinson 1996). As espécies de moscas mais intimamente associadas ao homem nos ambientes urbanos são representadas pelas famílias Calliphoridae, Sarcophagidae e Muscidae e, portanto, são consideradas as mais importantes sob o ponto de vista epidemiológico.

Os seus ovos são, geralmente, depositados em matérias orgânicas de origem vegetal e/ ou animal em decomposição por fermentação ou putrefação, lixo de origem doméstica, restaurantes, lixões a céu aberto e terrenos baldios, locais propícios ao desenvolvimento de suas larvas. Por serem insetos ectotérmicos possuem influência direta dos fatores climatológicos (Mello et al. 2007) e, em climas mais quentes o desenvolvimento e a ovogênese tendem a acelerar, propiciando o sincronismo de numerosas gerações diferentes, o que aumenta rapidamente as populações de moscas, e consequentemente e facilita sua dispersão e a invasão domiciliar.

Mais de 65 agentes patogênicos para o homem e animais domésticos podem ser veiculados 
pelos califorídeos, como: vírus, bactérias, fungos, protozoários e helmintos, além de alergias, e os processos de miíases primárias, secundárias e pseudomiíases (Greenberg 1971; Guimarães et al. 1983; Furlaneto et al. 1984; Guimarães e Papavero, 1999; Milward de Azevedo e Carraro 2000). Do ponto de vista epidemiológico, as espécies de Chrysomya causam preocupação, dada a sua capacidade de dispersão, densidade populacional, diversificação, hábito alimentar e, principalmente, pelo fato de transportar patógenos (Greenberg 1971).

\section{Material e Métodos}

\section{Local de coleta:}

O estudo foi realizado em dois ambientes distintos (urbano e rural) de amostragem no município de Vassouras, estado do Rio de Janeiro (latitude: $22^{\circ} 24^{\prime} 14^{\prime \prime} \mathrm{S}$; longitude: $43^{\circ}$ 39' 45" O e altitude: $416,822 \mathrm{~m}$. Os referidos pontos compreenderam o bairro Parque Cacique (bairro tipicamente residencial, e por isso caracterizado como ambiente urbano), e a estrada de Barão de Vassouras (caracterizada como ambiente rural pela presença de grande vegetação, além de ser cortada por um rio).

O Parque Cacique (latitude: $22^{\circ} 23^{\prime} 53^{\prime \prime} \mathrm{S}$ - longitude: $43^{\circ} 38^{\prime} 43^{\prime \prime} \mathrm{O}$ ), é um bairro residencial, e está localizado a aproximadamente $1,97 \mathrm{Km}$ do centro da cidade, e é considerado neste estudo como uma área urbana. Nas imediações do ponto de coleta há vegetação secundária de mata atlântica, com grande variedade de árvores frutíferas. No local ainda há a presença de pássaros e animais domésticos e de latões de lixo residencial a cerca de $9 \mathrm{~m}$ de distância das armadilhas de captura dos insetos.

A estrada Barão de Vassouras (latitude: $22^{\circ} 23^{\prime} 25^{\prime \prime}$ S- longitude: 43 40'47'O), situa-se na periferia da cidade, a cerca de $2 \mathrm{~km}$ do centro e foi considerada neste estudo como área rural. A área possui abundante vegetação secundária de mata atlântica, plantas frutíferas, presença de animais domésticos e várias espécies de pássaros, uma mina d'água, e distante, cerca de 700m do Rio das Cruzes (Fonte: Prefeitura de Vassouras, RJ).

\section{Coleta e identificação:}

A captura das moscas foi realizada por uso de armadilhas semelhantes à proposta por Ferreira (1978) durante o período de novembro de 2005 a outubro de 2006. Foram realizadas duas coletas semanais, rigorosamente realizadas nas três primeiras semanas de cada mês, entre o horário de $7 \mathrm{~h}$ e $8 \mathrm{~h}$ As capturas dos dípteros foi realizadas com armadilhas adaptadas daquelas descritas por Ferreira (1978). A armadilha (Figura 1) foi constituída por uma lata pintada de preto com $10 \mathrm{~cm}$ de diâmetro e $12 \mathrm{~cm}$ de altura, contendo quatro aberturas no terço inferior para a entrada dos insetos. Na abertura superior da lata foi colocado um cone de tela de náilon para impedir que as moscas escapassem. Sobre o cone e preso à borda superior da lata foi fixado com elástico um plástico para recolher as moscas. No interior de cada armadilha foi colocado um recipiente de plástico contendo $50 \mathrm{~g}$ de isca, à base de vísceras de ave. Cada armadilha foi suspensa cerca de 1,5 $\mathrm{m}$ do solo utilizando-se, para isso, quatro pedaços de fio de náilon. A temperatura e a umidade dos locais de coleta foram registradas diariamente com a utilização de termo 
higrômetro.

As moscas coletadas foram eutanasiadas com éter, deixadas secar e identificados apenas os califorídeos, que foram acondicionados em recipientes apropriados contendo álcool $70 \%$ e mantidos à temperatura de $15^{\circ} \mathrm{C}$ no Laboratório de Insetos Vetores Campo Experimental a serem processados posteriormente. Para a identificação das moscas foi utilizada a chave Mello (2003), como ferramenta de precisão ao diagnóstico do material em análise e realizada com o auxílio de microscópio estereoscópio, sistematicamente de acordo com cada coleta, no Laboratório de Zoologia no Conjunto Universitário, onde foi depositado. Um exemplar de cada espécie de todo o material coletado foi preservado para a coleção entomológica do Laboratório de Insetos Vetores do Unidade de Ensino, Pesquisa e Extensão Prof Antonio Orlando Izolani, CampusCampo Experimental/USS.

\section{Tratamento dos dados:}

Para avaliar e comparar as diferenças na composição populacional dos dois ambientes de coleta foram utilizados o Índice de Diversidade de Shannon-Winer, o Índice de Dominância de Simpson (ZAR, 1999), o Índice de Riqueza de Espécies de Jackknife, o Índice de Equitabilidade de Hill e o Índice de correlação de Sperman a 5\% de probabilidade (Zar, 1999), este último, utilizado para fornecer os coeficientes de correlação (rs) entre as flutuações populacionais dos califorídeos coletados e as temperaturas máximas, mínimas e médias. As diferenças entre as duas populações estudadas foram analisadas pelo teste não paramétrico de Mann-Whitney a 5\% de probabilidade. O teste estatístico utilizado para a identificação da normalidade das variáveis foi o teste D'Agostino a 5\% de significância. A escolha de testes não paramétricos para análise dos dados deve-se ao fato de estes não satisfazerem aos parâmetros exigidos pelos testes paramétricos. Para as análises estatísticas foram utilizados os programas BioEstat 5.0 e DivEs - Diversidade de espécies (Rodrigues, 2005).

\section{Resultados}

Das 2185 moscas coletadas, a família Calliphoridae: Chrysomya megacephala (Fabricius, 1 805), Chrysomya albiceps (Wilddemann, 1 830), Phaenicia eximia (Wiedmann, 1819 ), Phaenicia cuprina (Wilddemann, 1 830), Cochliomyia macelaria (Fabricius, 1 805), se destacou das demais, com 1421 indivíduos, (65, 03\%) seguida da família Sarcophagidae com 743 indivíduos (34\%), e Muscidae (Musca domestica Linnaeus, 1 758) com 21 indivíduos coletados $(<1,0 \%)$ (Tabela 1$)$.

No ambiente rural, a família Calliphoridae (70,70\%) apresentou o maior percentual de espécimes coletados, seguida da família de Sarcophagidae (28,45\%). No ambiente urbano, também a família Calliphoridae $(59,64 \%)$ obteve o maior percentual de moscas capturadas, seguida de Sarcophagidae (39,29\%). A família Muscidae mostrou-se pouco representativa e correspondendo a aproximadamente $1 \%$ dos dípteros coletados em ambas as áreas de amostragem (Tabela 1).

As espécies de califorídeos mais coletadas na área rural foram C. albiceps, com 279 espécimes $(26,20 \%)$ e C. megacephala, com 276 espécimes (25,92\%), seguidas de 
P. eximia, com 140 espécimes $(13,15 \%), P$. cuprina com 35 espécimes $(3,29 \%)$ e $C$. macellaria, com 23 espécimes (2,16\%) (Tabela 1).

Na área urbana, a espécie mais numerosa foi C. megacephala, com 231 espécimes (20,63\%), seguida de $P$. eximia, com 180 espécimes (16,07\%); C. albiceps com 162 espécimes (14,46\%), C. macellaria com 48 espécimes (4,29\%) e P. cuprina, com 47 espécimes $(4,20 \%)$. As diferenças entre as populações (rural e urbana) não foram significativas no teste de Mann-Whitney ( $\mathrm{P}=0,4584)$, no entanto, o índice de diversidade de ShannonWiner foi maior no ambiente urbano, e o índice de dominância de Simpson, no rural. A riqueza de espécies e a equitabilidade também foi maior no ambiente urbano (Tabela 1).

Tabela 1: Número total, percentual e Índice de Diversidade de Shannon-Wiener dos dípteros muscóoides coletados no período de outubro de 2005 a novembro de 2006, no município de Vassouras - RJ.

\begin{tabular}{|c|c|c|c|c|c|c|c|c|}
\hline & \multicolumn{5}{|c|}{ Ambientes } & & & \\
\hline & \multicolumn{2}{|c|}{ Rural } & & \multicolumn{2}{|c|}{ Urbano } & & \multicolumn{2}{|c|}{ Total } \\
\hline & $\mathrm{n}$ & $\%$ & & $\mathrm{n}$ & $\%$ & & $\mathrm{n}$ & $\%$ \\
\hline C. megacephala & 276 & 25.92 & & 231 & 20.63 & & 507 & 23.20 \\
\hline C. albiceps & 279 & 26.20 & & 162 & 14.46 & & 441 & 20.18 \\
\hline p. eximia & 140 & 13.15 & & 180 & 16.07 & & 320 & 14.65 \\
\hline P. cuprina & 35 & 3.29 & & 47 & 4.20 & & 82 & 3.75 \\
\hline Co.macellaria & 23 & 2.16 & & 48 & 4.29 & & 71 & 3.25 \\
\hline Calliphoridae & 753 & 70.70 & & 668 & 59.64 & & 1421 & 65.03 \\
\hline Sarcophagidae & 303 & 28.45 & & 440 & 39.29 & & 743 & 34.00 \\
\hline M. domestica & 9 & 0.85 & & 12 & 1.07 & & 21 & 0.96 \\
\hline Total & 1065 & 100.00 & 0 & 1120 & 100.00 & 0 & 2185 & 100.00 \\
\hline $\begin{array}{l}\text { Índice de } \\
\text { Diversidade }\end{array}$ & \multicolumn{2}{|c|}{0.564} & & \multicolumn{2}{|c|}{0.625} & & & \\
\hline $\begin{array}{l}\text { Índice de } \\
\text { Dominância }\end{array}$ & \multicolumn{2}{|c|}{0.735} & & \multicolumn{2}{|c|}{0.602} & & & \\
\hline $\begin{array}{c}\text { Riqueza de } \\
\text { Espécies (Smax) }\end{array}$ & \multicolumn{2}{|c|}{5.000} & & \multicolumn{2}{|c|}{7.500} & & & \\
\hline Equitabilidade & \multicolumn{2}{|c|}{2.963} & & \multicolumn{2}{|c|}{3.275} & & & \\
\hline
\end{tabular}


Ao correlacionar a presença de Calliphoridae com a temperatura durante o período de coleta no município de Vassouras prevaleceu o aparecimento de $P$. eximia $(46 \%) \mathrm{em}$ temperaturas compreendidas entre $15^{\circ} \mathrm{C}$ a $20^{\circ} \mathrm{C}, \mathrm{C}$. megacephala $(44 \%)$ entre $21^{\circ} \mathrm{C}$ e $25^{\circ}$ e C. albiceps (50\%) entre $26^{\circ} \mathrm{C}$ e $30^{\circ} \mathrm{C}$ (Tabela 2$)$.

Tabela 2: Frequência populacional dos califorídeos, de acordo com a temperatura média mensal, no período de novembro de 2005 a outubro de 2006, no município de Vassouras - RJ.

\begin{tabular}{|c|c|c|c|c|c|c|}
\hline & \multicolumn{2}{|c|}{$15^{\circ} \mathrm{C}$ a $20^{\circ} \mathrm{C}$} & \multicolumn{2}{|c|}{$21^{\circ} \mathrm{C}$ a $25^{\circ} \mathrm{C}$} & \multicolumn{2}{|c|}{$26^{\circ} \mathrm{C}$ a $30^{\circ} \mathrm{C}$} \\
\hline & $\mathbf{n}$ & $\%$ & $\mathbf{n}$ & $\%$ & $\mathbf{n}$ & $\%$ \\
\hline C. megacephala & 86 & 23 & 311 & 44 & 110 & 32 \\
\hline C. albiceps & 69 & 18 & 202 & 29 & 170 & 50 \\
\hline P. eximia & 172 & 46 & 111 & 16 & 37 & 11 \\
\hline P. cuprina & 39 & 11 & 31 & 4 & 12 & 3 \\
\hline Co. macellaria & 8 & 2 & 50 & 7 & 13 & 4 \\
\hline Total & \multicolumn{2}{|c|}{374} & \multicolumn{2}{|c|}{705} & \multicolumn{2}{|c|}{342} \\
\hline
\end{tabular}

No entanto, na correlação de Sperman, somente C. megacephala e C. albiceps apresentaram relação significativamente positiva com a temperatura mínima $(\mathrm{P}=0,0208$ e 0, 0043), respectivamente (Tabela 3 ).

Tabela 3: Coeficiente de Correlação de Sperman entre parâmetros climáticos do dia da coleta com o número de indivíduos da família Calliphoridae coletados no período de outubro de 2005 a novembro de 2006, no município de Vassouras -RJ.

\begin{tabular}{|c|c|c|c|c|c|}
\hline & C. megacephala & C. albiceps & P. eximia & P. cuprina & C.macellaria \\
\hline & \multicolumn{5}{|c|}{ Temperatura Máxima } \\
\hline Coeficiente de Spearman $(r s)=$ & 0.320 & 0.515 & -0.458 & 0.469 & 0.232 \\
\hline \multirow[t]{2}{*}{ probabilidade $=$} & 0.311 & 0.086 & 0.134 & 0.125 & 0.468 \\
\hline & \multicolumn{5}{|c|}{ Temperatura Mínima } \\
\hline Coeficiente de Spearman $(r s)=$ & 0.655 & 0.757 & -0.042 & 0.147 & -0.027 \\
\hline \multirow[t]{2}{*}{ probabilidade $=$} & $0.021^{\mathrm{s}}$ & $0.004^{s}$ & 0.896 & 0.647 & 0.934 \\
\hline & \multicolumn{5}{|c|}{ Temperatura Média } \\
\hline Coeficiente de Spearman $(r s)=$ & 0.472 & 0.570 & -0.310 & 0.352 & 0.169 \\
\hline \multirow[t]{2}{*}{ probabilidade $=$} & 0.121 & 0.053 & 0.327 & 0.262 & 0.600 \\
\hline & \multicolumn{5}{|c|}{ Amplitude Térmica } \\
\hline Coeficiente de Spearman $(r s)=$ & -0.4398 & -0.4398 & -0.4185 & -0.3617 & -0.0572 \\
\hline probabilidade $=$ & 0.1525 & 0.1525 & 0.1757 & 0.2479 & 0.8597 \\
\hline
\end{tabular}

$\mathrm{s}=$ Correlação significativa 
A temperatura média no município de Vassouras durante o período de estudo ficou entre $16^{\circ} \mathrm{C}$ e $28^{\circ} \mathrm{C}$. O maior número de califorídeos coletados ocorreu a temperaturas compreendidas entre $21^{\circ} \mathrm{C}$ e $25^{\circ} \mathrm{C}$ nos meses de janeiro a abril, representando $50 \%$ dos 705 espécimes de Calliphoridae capturados em ambas as áreas de estudo (Figura 2).

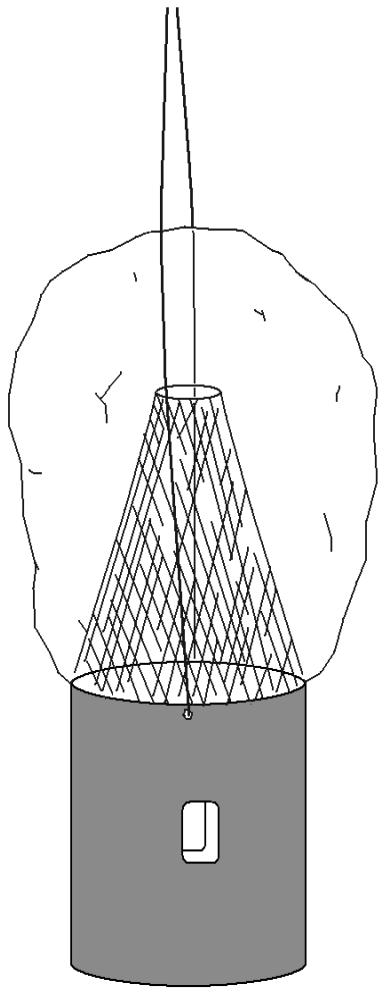

Figura 1. Desenho esquemático da armadilha utilizada neste estudopara a captura de dípteros muscóoides. 
Ocorrência de Dípteros Muscóides (Calliphoridae) no Município de Vassouras, RJ

Raquel S. Macedo - Vinicius M. Carraro - Cleber B. Espindola - Marise M. O. Cabral

A

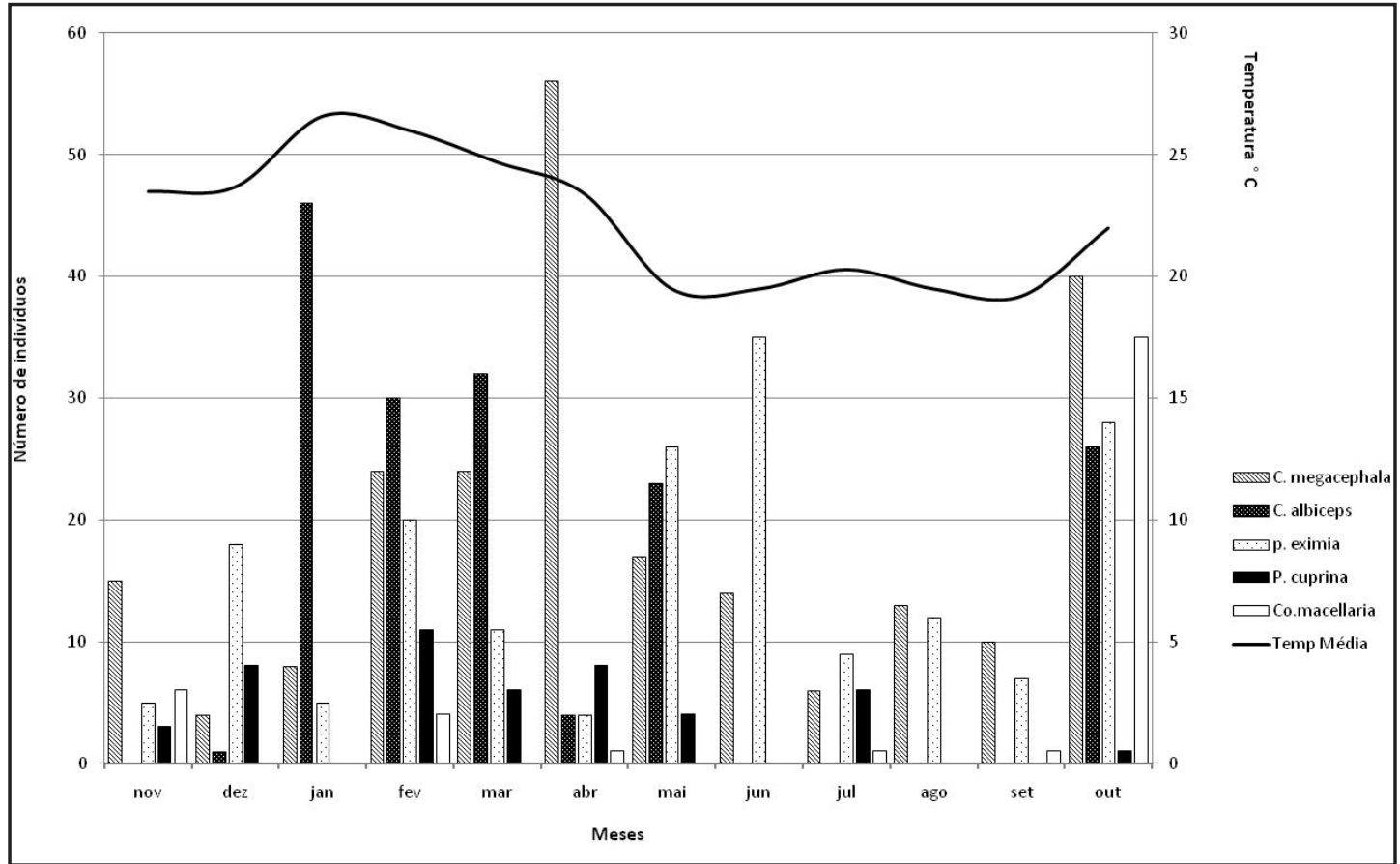

B

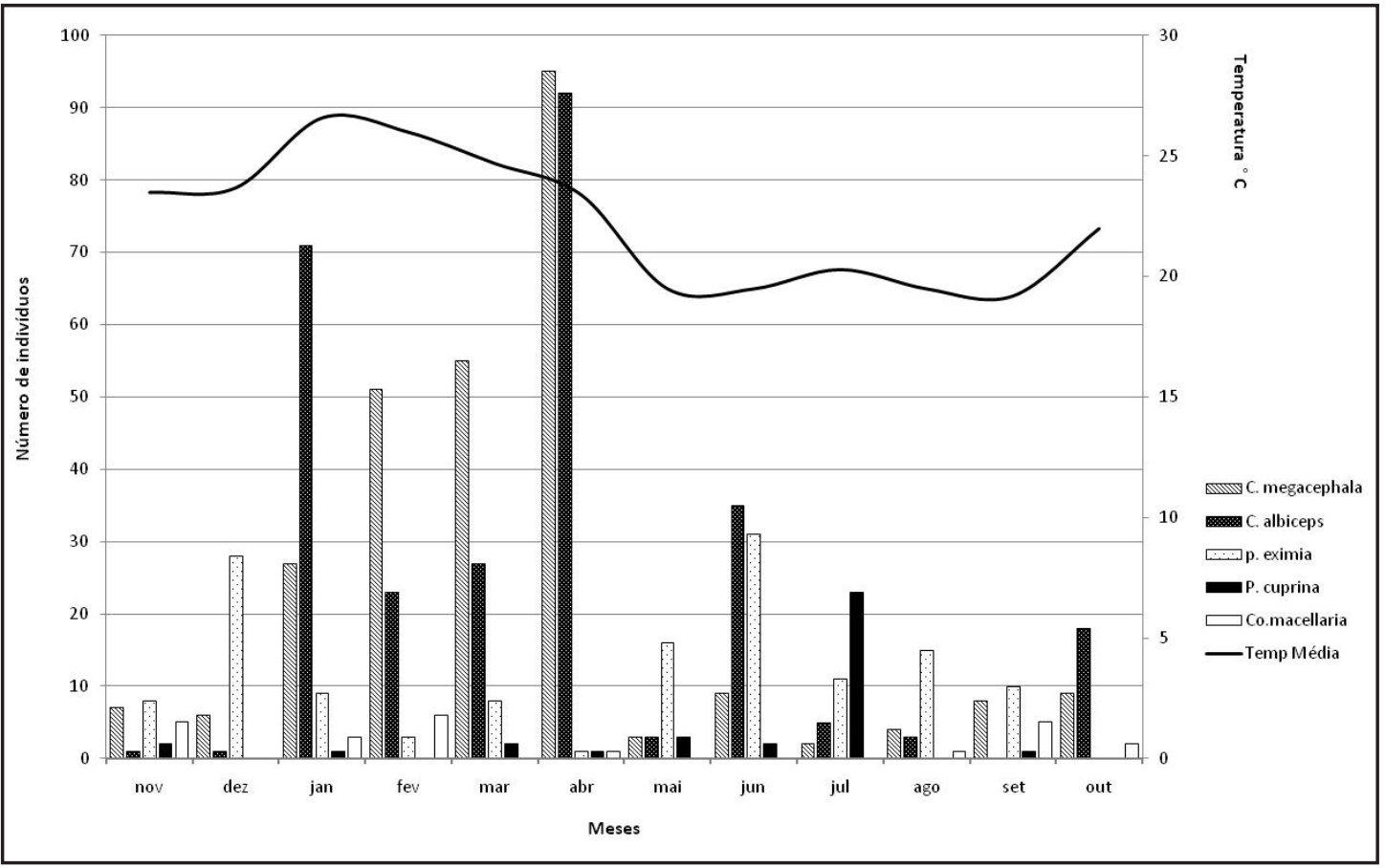

Figura 2. Temperatura média $\left({ }^{\circ} \mathrm{C}\right)$ e número total das espécies de califorídeos coletadas na área rural (A) e urbana (B), durante o período de novembro de 2005 a outubro de 2006 no município de Vassouras - RJ. 


\section{Discussão e Conclusão}

Neste estudo destacou-se a presença da família Calliphoridae tanto nas áreas rural urbana do município de Vassouras - RJ. A transformação do ambiente natural em urbano e rural ocorrida nas áreas de coleta influenciou a mudança da flora e fauna locais do município em estudo (observação pessoal). Pensa-se que a ocorrência de espécies silvestres em áreas urbanizadas esteja associada aos desflorestamentos em matas próximas, levandoas a tentarem se colonizar nesses novos ambientes, o que tem ocorrido constantemente [d'Almeida e Braga, 2007]. São conhecidas cerca de 150 mil espécies de moscas ( 70\% do total da ordem Diptera), que vivem praticamente em todos os ambientes do mundo e cujas larvas e adultos consomem vários tipos de alimento: sangue, excrementos, carne em decomposição, madeira, frutas e néctar (Gomes e Von Zuben 2005). Os califorídeos têm demonstrado grande capacidade de adaptação às condições ecológicas criadas pelo homem no processo de urbanização [Ferreira e Lacerda 1993]. Esta família apresenta também espécies de dispersão contínua, atraídas por fontes de criação como matadouros, leiterias, depósitos de lixo, além de outras, localizadas próximas às cidades (Quaterman et al. $1954 \mathrm{a}, \mathrm{b})$.

As espécies $C$. albiceps e C. megacephala, foram as moscas mais coletadas na Estrada Barão - Vassouras (ambiente rural) (26,20\% e 25,92 \%, respectivamente). Estes dados corroboram com Mello et al (2004), pois mostra a presença destas duas espécies como as mais bem representadas em coletas realizadas em uma área rural (sítio) do município de Paracambi - RJ. Os mesmo autores detectaram a prevalência de C. megacephala $(23,20 \%)$ em relação a $C$. albiceps $(20,18 \%)$, da mesma forma que os resultados deste estudo. D' Almeida e Mello (1996) realizaram captura de dípteros muscóides com utilização de atrativos como iscas de peixe (sardinha), fígado (bovino), carne (bovina moída), camarão (sem casca), lula, banana fermentada e observaram que a espécie C. megacephala possui preferência em $99,9 \%$ para a carne bovina.

Detectou-se a presença maior de $C$. megacephala, $P$. eximia e $C$. albiceps no ambiente urbano e que podem ter sido atraídas pela presença de dois latões para coleta de lixo domiciliar. A presença do lixo doméstico propicia o aparecimento da mosca varejeira [Ferreira e Lacerda 1993; Linhares 1981 a, b; Ribeiro e Prado 1986; Queiroz 1986], o que confirma a presença de $C$. megacephala em locais ricos em matéria orgânica [Lima e Luz 1991], e demonstra sua importância na transmissão de enterobactérias. Tanto estas espécies de moscas como a presença de P. eximia, P. cuprina e C. macellaria na área urbana também foram registradas por Mello et al. (2004) na região urbana do município de Paracambi - RJ.

Os resultados sugerem que $C$. megacephala foi a espécie que apresentou maior capacidade de dispersão e adaptação nos dois ambientes estudados, distribuindo-se praticamente na mesma proporção nas duas áreas de coleta. Este dado confirma que C. megacephala é um inseto sinantrópico de ampla distribuição, tendo sido observadas na região de Paracambi em $+49,01 \%$ (Mello et al. 2004), em Seropédica (Carraro e Milward-de-Azevedo 1999 a, b), na zona norte do Rio de Janeiro [Lopes, 2000] chegando até o estado do Amazonas (Baumgartner e Greenberg 1984). O gênero Chrysomya, aliás, é tipicamente sinantrópico (Mello et al. 2007).

O aumento populacional de C. megacephala em temperaturas compreendidas entre $21^{\circ} \mathrm{C}$ 
e $25^{\circ} \mathrm{C}$ corrobora com Souza e Linhares (1997), que assinalaram o pico populacional das espécies de Chrysomya no Brasil, nos meses com temperaturas superiores a $18^{\circ} \mathrm{C}$. Assim como fora demonstrado que a influência da temperatura e da precipitação pluviométrica na sazonalidade de $C$. megacephala, culmina com maiores capturas nos meses quentes e chuvosos no município de Presidente Prudente - SP (Dias et al. 2009). Esse comportamento indica que as temperaturas mais baixas, por se tratar de espécies introduzidas (Oliveira 1982), associadas à diminuição da oferta alimentar, constituem fator limitante para esses dípteros.

Entre os califorídeos, C. megacephala foi a espécie mais abundante no município de Vassouras - RJ, com distribuição homogênea entre as áreas rural e urbana. A maior abundância dos dípteros Calliphoridae foi registrada na área rural. A estreita ligação da área urbana com a rural e o índice crescente do volume de matéria orgânica acumulada pelo homem têm proporcionando o aumento significativo da população de dípteros muscóides na região. Os dados obtidos servirão como base para a avaliação do papel destes dDípteros na veiculação de estruturas parasitárias de interesse médico e veterinário no município de Vassouras. 


\section{Referências}

Baumgartner, D. L. and Greenberg, B. (1984). The genus Chrysomya (Diptera, Calliphoridae) in the New World. Journal of Medical Entomology 21:105-113.

Carraro, V. M. and Milward-de-Azevedo, E. M. V. (1999a). Quantitative description of Calliphoridae dipterans captured on the Campus of the Federal Rural University of Rio de Janeiro using sardine bait. Revista Brasileira de Zoologia 51: 307-11.

Carraro, V. M. and Milward-de-Azevedo, E. M. V. (1999b). Quantitative description of Calliphoridae dipterans captured on the Campus of the Federal Rural University of Rio de Janeiro using sardine bait. Revista Brasileira Zoociências 1 (1): 77-89.

D’ Almeida, J. M. and Mello, R. P. (1996). Comportamento de dípteros muscóides frente a substratos de oviposição em laboratório, no Rio de Janeiro, RJ. Brasil. Memórias do Instituto Oswaldo Cruz 91 (1): 137-138.

D’ Almeida, J. M. and Fraga, M.B, (2007). Efeito de diferentes iscas na atração de califorídeos (Diptera) no campus do Valonguinho, Universidade Federal Fluminense, Niterói, RJ, Brasil. Revista Brasileira de Parasitologia Veterinária, 16 (4): 199-204

Dias L. S., Santarém, V.A., Almeida, M.S.R., Medina, A.O., da Silva, A.V. (2009). Biodiversidade de Moscas Calliphoridae no Lixão Urbano de Presidente Prudente, São Paulo, Brasil. Arquivo do Instituto Biológico, São Paulo, 76, (4): 659-663.

Ferreira, M. J. M. and Lacerda, P. V. (1993). Muscóides sinantrópicos associados ao lixo urbano em Goiânia, Goiás. Revista Brasileira de Zoologia, 10 (2): 185-195.

Furlaneto, S. M. P.; Campos, M. L. C.; Hársi, C. M.; Buralli, G. M. and Ishiata, G. K. (1984). Microorganismos enteropatogênicos em moscas africanas pertencentes ao gênero Chrysomya (Diptera: Calliphoridae) no Brasil. Revista de Microbiologia 15 (3): 170-174.

Gomes, L. \& Von Zuben, C. (2005). O Novo Papel das Moscas. Revista Ciência Hoje, p: $70-72$.

Greenberg, B. (1991). Flies as Forensic Indicators. Journal of Medical Entomology 28 (5): 565-577.

Greenberg, B. (1971). Ecology, classification and biotic association. In: Greenberg, B. ed. Flies and diseases. Princeton: Princeton University Press, v.1, 856 p.

Guimarães, J. H. and Papavero, N. (1999). Myiasis in Man and animals in the Neotropical region. São Paulo: Ed. Plêiade/ FAPESP. 308 p.

Guimarães, J. H.; Papavero, N. and Prado, A. P. (1983). As milíases na região neotropical: identificação, biologia, bibliografia. Revista Brasileira de Zoologia 1: 239-416.

Lima, M. S. and Luz, E. (1991). Espécies exóticas de Chrysomya (Diptera: Calliphoridae) como veiculadoras de enterobactérias patogênicas em Curitiba, Paraná, Brasil. Acta Biológica Paranaense, Curitiba, 20 (1-4): 61-83.

Linhares, A. X. (1981a). Synantropy of Calliphoridae and Sarcophagidae (Diptera) in the city of Campinas, São Paulo, Brazil. Revista Brasileira de Entomologia 25: 189-215.

Linhares, A. X. (1981b). Synantropy of Muscidae, Fanniidae and Anthomyiidae (Diptera) in the city of Campinas, São Paulo, Brazil. Revista Brasileira de Entomologia 25 (4): 231-243. 
Mello, R. P.; Gredilha, R. and Neto, E. G. G. (2004). Dados preliminares sobre sinantropia de califorídeos (Diptera: Calliphoridae) no município de Paracambi, RJ. Revista da Universidade Rural, ser. Ci. Vida. Seropédica, RJ, EDUR, 24 (2): 97- 101.

Mello, R. P. (2003). Chave para identificação das formas adultas das espécies da família Calliphoridae (Diptera, Brachycera, Cyclorrapha) encontradas no Brasil. Entomologia \& Vetores 10 (2): 255-268.

Mello, R. P.; Queiroz, M. M. C. and Aquiar-Coelho, V. M. (2007). Population fluctuations of Calliphoridae species (Diptera, Calliphoridae) in the Biological Reserve of Tinguá, state of Rio de Janeiro, Brazil. Iheringia. Série Zoologia, Porto Alegre, 97(4): 481485 .

Milward-de-Azevedo, E. M. V. and Carraro, V. M. (2000). Controle biológico clássico contra pragas de interesse médico-veterinário e sanitário. Revista Brasileira Medicina Veterinária 22(1): 11-14.

Oliveira, C. M. B. (1982). Ocorrência e flutuação populacional de três espécies do gênero Chrysomya. Pesquisa Agropecuária Brasileira 17 (12): 1707-1708.

Quaterman, K. D.; Kilpatrick, J. N. and Mathis, W. (1954a). Fly dispersal in rural area near savannah, Georgia. Journal of Economy Entomology 47: 413-419.

Quaterman, K. D.; Kilpatrick, J. N. and Mathis, W. (1954b). Urban fly dispersal in the area of savannah, Georgia. Journal of Economy Entomology 47: 413-419.

Queiroz, S. M. P. (1986). Dípteros muscóides sinantrópicos (Calliphoridae, Muscidae, Fannidae e Sarcophagidae) em sete biótopos de Curitiba. Tese de Mestrado. Universidade Federal do Paraná, Curitiba, 188p.

Ribeiro, O. B. and Prado,A.P.(1986). Chrysomya megacephala (Fabricius, 1794) associada ao lixo urbano. Resumos do XII Congresso Brasileiro de Zoologia, Campinas.

Robinson, W. H. (1996). Urban entomology: insect and mite pests in the human environment. London: Chapman \& Hall, 430p.

Rodrigues, W. C. DivEs - Diversidade de espécies. Versão 2.o. Software e Guia do Usuário. 2005. Disponível em: <http://www.ebras.bio.br/dives>

Sordillo, C. M. O. (1991). Sinantropia e Análise da variação espacial no índice proposto por Nuorteva (1963) em dípteros muscóides no município do Rio de Janeiro, RJ, Brasil. Tese de Mestrado, Universidade Federal do Rio de Janeiro, 318 pp.

Souza, A. M. and Linhares, A. X. (1997). Diptera and Coleoptera of potential forensic importance in southeastern Brazil: relative abundance e seasonality. Medical and Veterinary Entomology 11: 269-283.

Zar, J. H. (1999). Bioestatistical Analyses 4th ed. New Jersey, Prentice Hall. 663p. 